\title{
Fusión del tercer molar inferior con un cuarto molar. Reporte de tres casos.
}

\section{Fusion of the lower third molar with a fourth molar. Report of three cases.}

\author{
Enrique Darío Amarillas Escobar*
}

\section{RESUMEN}

Los dientes supernumerarios y la fusión dental son anomalías del desarrollo dental cuyas causas aún no se han dilucidado con certeza. El cuarto molar inferior, también denominado distomolar, es uno de los dientes supernumerarios con menor frecuencia de aparición clínica y su fusión con el tercer molar es una condición todavía menos común. A continuación, se reportan los casos clínicos de tres pacientes masculinos que presentaron fusión del tercer molar inferior derecho con un distomolar tratados mediante odontectomía.

Palabras clave: Fusión dental, diente supernumerario, cuarto molar.

\section{INTRODUCCIÓN}

$\mathrm{D}$ urante la odontogénesis se puede presentar una amplia variedad de alteraciones respecto al número, tamaño, forma, posición, color y estructura de los órganos dentarios.

La fusión dental es una de estas anomalías del desarrollo dental que consiste en la unión de dos gérmenes dentarios contiguos. Esta fusión puede ser completa o incompleta dependiendo del estadio en que se presenta dicha unión. Cuando ocurre antes de la calcificación, la fusión implica todos los componentes de los órganos dentarios incluyendo esmalte, dentina, cemento y pulpa, resultando en un solo órgano dentario de tamaño cercano a lo normal. Si la unión se produce en una etapa más tardía, los órganos dentarios involucrados pueden
ABSTRACT

Supernumerary teeth and dental fusion are abnormalities of dental development whose causes have not yet been elucidated with certainty. The lower fourth molar, also called distomolar, is one of the supernumerary teeth with the least frequency of clinical appearance and its fusion with the third molar is an even less common condition. Next, the clinical cases of three male patients who presented fusion of the right lower third molar with a distomolar treated by odontectomy are reported.

Keywords: Dental fusion, supernumerary tooth, fourth molar.

\footnotetext{
* Cirujano Maxilofacial. Profesor de asignatura de la Licenciatura de Cirujano Dentista de la Facultad de Estudios Superiores Zaragoza de la Universidad Nacional Autónoma de México. Profesor asociado de la Licenciatura de Estomatología de la Universidad Autónoma Metropolitana Unidad Xochimilco.

Recibido: 17 de agosto de 2020. Aceptado: 26 de enero de 2021.

Citar como: Amarillas EED. Fusión del tercer molar inferior con un cuarto molar. Reporte de tres casos. Rev ADM. 2021; 78 (2): 95-99. https://dx.doi. org/10.35366/99285
}

tener las coronas separadas y la fusión estar limitada a las raíces, siendo mayor el tamaño del órgano dentario. ${ }^{1,2}$ Las cámaras pulpares y los conductos radiculares pueden entonces ser independientes o estar comunicados. ${ }^{3,4}$

La etiología de la fusión dental es desconocida, se han descrito como causas probables la predisposición genética, las diferencias raciales y la impactación traumática entre sí de los gérmenes dentarios involucrados durante su desarrollo., 4

La frecuencia de fusión dental es extremadamente baja, siendo tan sólo del $0.1 \%$ en la dentición permanente y del $0.5 \%$ en la dentición temporal, ${ }^{4}$ y se presenta con igual oportunidad en hombres y mujeres. ${ }^{3}$ Esta anomalía es más común en la región dental anterior de ambos maxilares, estando involucrados dos órganos dentarios normales, por lo que la fusión de un órgano dentario 
normal con uno supernumerario es una condición aún menos frecuente. ${ }^{3,6}$

A continuación, se describen tres casos clínicos de terceros molares inferiores derechos fusionados con distomolares.

\section{PRESENTACIÓN DEL CASO}

Caso 1. Varón de 16 años de edad, aparentemente sano, referido por ortodoncista para manejo quirúrgico preortodóntico por hallazgo radiográfico consistente en una posible fusión del tercer molar inferior derecho y un cuarto molar, ambos en desarrollo coronal (Figura 1).

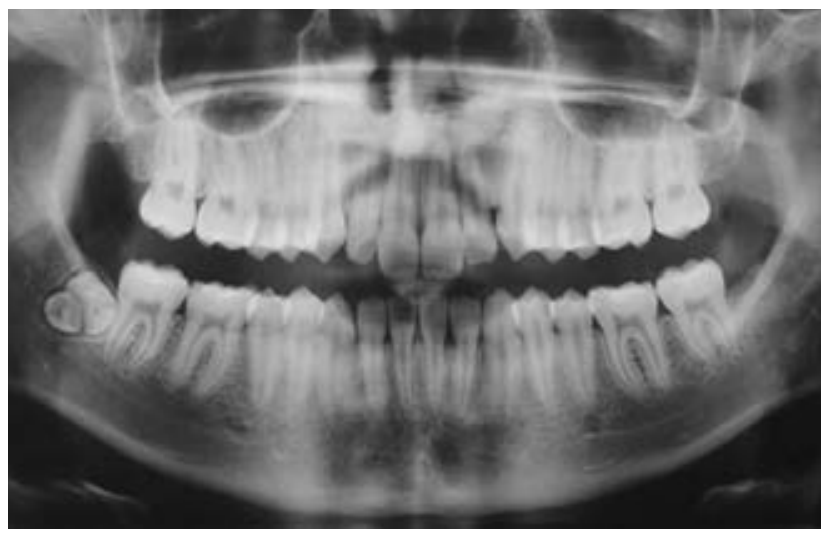

Figura 1: Imagen radiográfica de la fusión del tercer y cuarto molar en desarrollo.

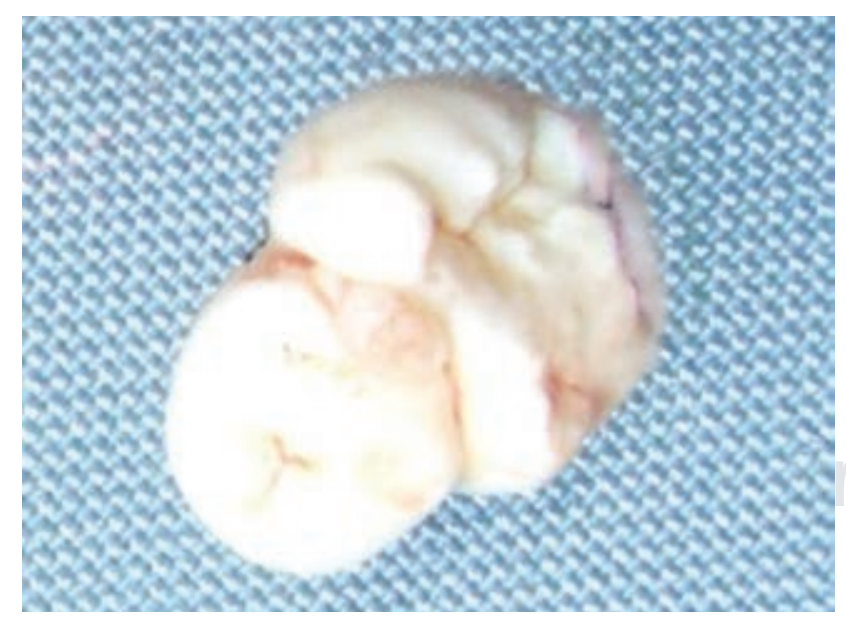

Figura 2: Presentación dimensional aproximada de los órganos dentarios fusionados, con giroversión mesial del tercer molar y el distomolar adyacente a la tabla ósea vestibular.

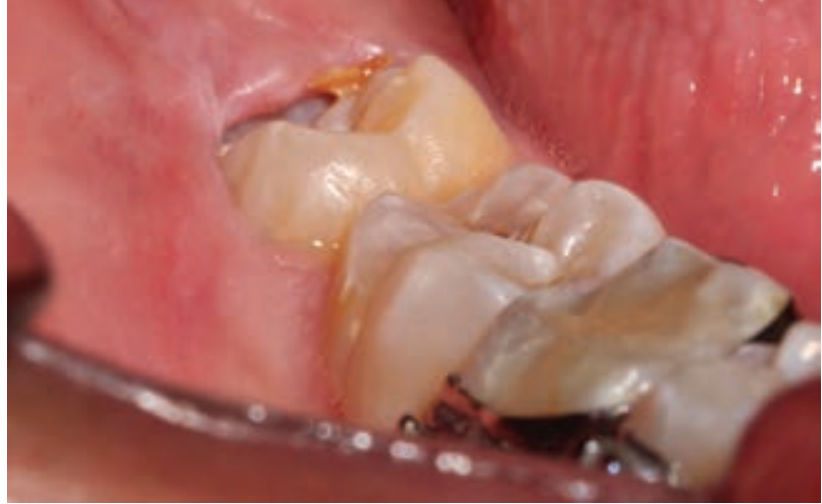

Figura 3: Imagen clínica de la fusión dental entre el tercer y cuarto molar asociada con pericoronitis.

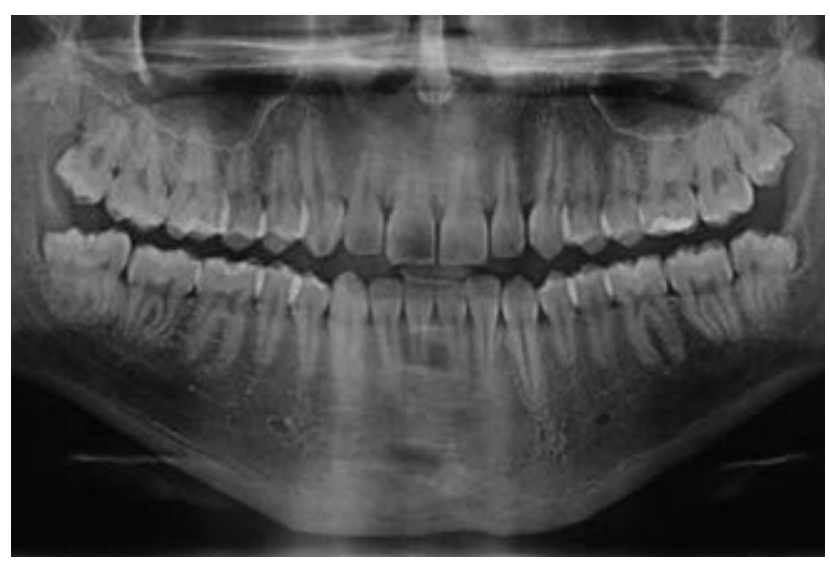

Figura 4: Detalle radiográfico del tercer y cuarto molar fusionados.

Destacó además agenesia del resto de los terceros molares. Se realizó odontectomía bajo anestesia local con técnica convencional, confirmando la fusión de estos órganos dentarios. El distomolar se encontraba fusionado en la superficie distal de la corona del tercer molar; sin embargo, éste último presentaba una giroversión mesial, por lo que el diente supernumerario estaba ubicado adyacente a la tabla ósea vestibular (Figura 2).

Caso 2. Hombre de 22 años de edad, quien acude a la Clínica Universitaria de Atención a la Salud (CUAS) Estado de México de la Facultad de Estudios Superiores Zaragoza (FES-Z) de la Universidad Nacional Autónoma de México (UNAM) por presentar episodios repetitivos de pericoronitis asociada al tercer molar inferior derecho parcialmente erupcionado. Al interrogatorio sin antecedentes médicos y dentales de importancia. Al realizar la exploración física intrabucal se observó que dicho órgano 
dentario presentaba macrodoncia y morfología irregular, por lo que se sospechó de fusión con un distomolar (Figura 3), lo cual se corroboró con la evaluación radiográfica (Figura 4) y del espécimen quirúrgico postodontectomía (Figura 5). El cuarto molar se encontraba fusionado en el aspecto distal del tercer molar y la unión se presentaba a nivel coronal y radicular compartiendo la misma cámara pulpar (Figura 6).

Caso 3. Varón de 17 años de edad, sin datos clínicos contribuyentes, referido para manejo quirúrgico previo a tratamiento ortodóntico consistente en odontectomía de terceros molares. Al evaluar la imagen radiográfica del tercer molar inferior derecho se observó que presentaba una morfología coronal inusual, por lo que se estableció el diagnóstico presuntivo de fusión con un diente supernumerario (Figura 7), mismo que fue corroborado tras la odontectomía. El cuarto molar se encontraba fusionado en el aspecto distolingual del tercer molar (Figura 8).

\section{DISCUSIÓN}

Los dientes supernumerarios son órganos dentarios adicionales de las denticiones fisiológicas primaria y per- manente. Esta alteración del desarrollo dental también se conoce como hiperdoncia. ${ }^{2}$

Su frecuencia de presentación varía entre poblaciones, en México el intervalo es del 2.8 al 5.6\%. ${ }^{7-10}$ Son más comunes en el sexo masculino que en el femenino con una relación de 2:1 y mucho más frecuentes en la dentición permanente que en la decidua. ${ }^{2}$ Pueden presentarse de manera aislada o ser múltiples y asociarse o no a condiciones como displasia cleidocraneal, síndrome de Gardner, síndrome de Crouzon, síndrome de Ehlers-Danlos, enfermedad de Fabry, fisura labioalveolo-palatina, entre otras. ${ }^{11}$

Los dientes supernumerarios más frecuentes son los mesiodens $(50 \%)$, los cuartos molares o distomolares superiores (25\%), los paramolares superiores (15\%) y los premolares inferiores $(3 \%){ }^{2}$

Morfológicamente pueden ser idénticos a los órganos dentarios normales (eumórficos o suplementarios) o bien tener una morfología anormal (heteromórficos), presentando microdoncia con una forma conoide, de clavija, tuberculada, infundibular o molariforme. Sólo el 25\% se encuentran erupcionados completa o parcialmente. ${ }^{2}$

Se desconoce el mecanismo exacto de la formación de los dientes supernumerarios, sugiriéndose diversos
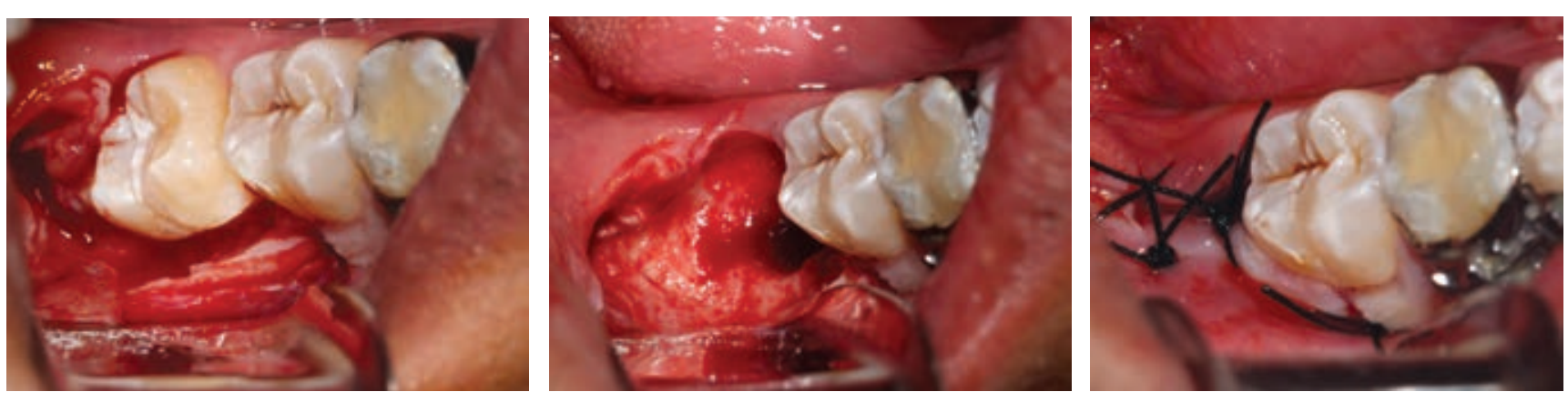

Figura 5: Técnica quirúrgica de odontectomía.
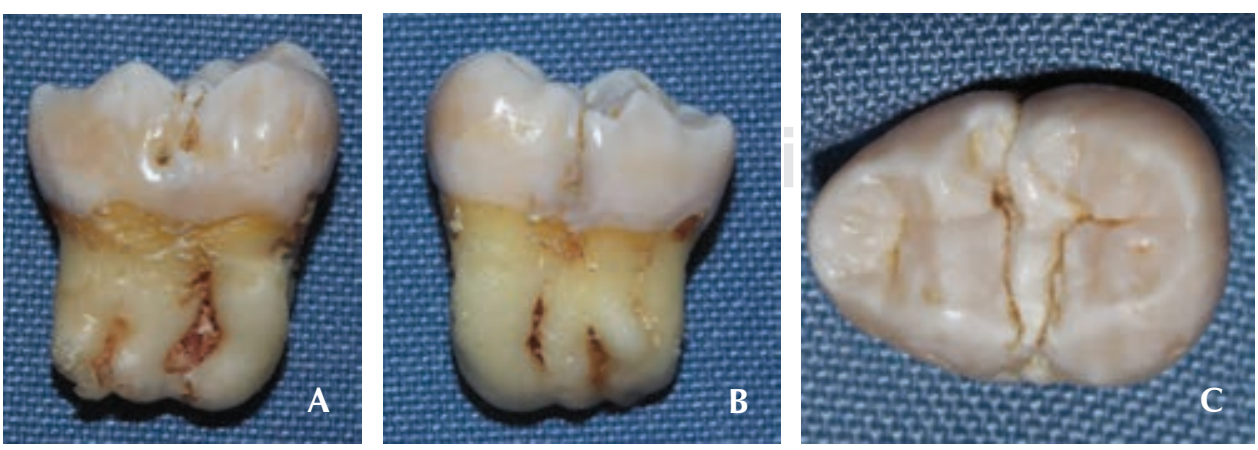

Figura 6:

Pieza quirúrgica. A) Aspecto vestibular. B) Vista lingual. C) Cara oclusal. 


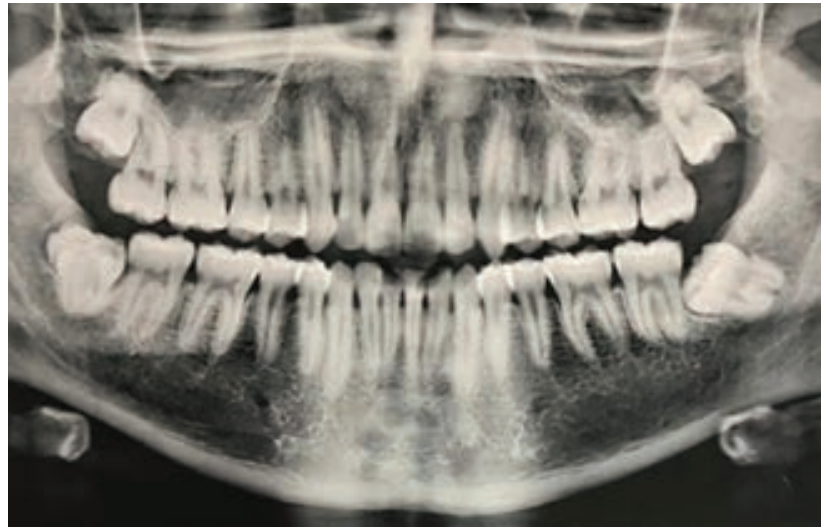

Figura 7: Presentación imagenológica de los órganos dentarios fusionados.

factores etiológicos, entre ellos la herencia y posibles desordenes del desarrollo dental como la sobreactividad de la lámina dental o la división del germen dentario, entre otros. ${ }^{12,13}$

El cuarto molar, también llamado distomolar o retromolar por su localización posterior a los terceros molares, ${ }^{14}$ es un diente supernumerario que en la mandíbula se presenta sólo en el 1\% de los casos. ${ }^{2}$

En este caso particular, los cuartos molares probablemente tienen su origen a partir de un crecimiento distal de la lámina dental. ${ }^{2}$ Su erupción es poco habitual. ${ }^{15}$

Su forma y tamaño es variable, aunque generalmente presentan microdoncia y una morfología similar a la de un molar inferior. ${ }^{14,16}$

Con poca frecuencia un distomolar se fusiona con el tercer molar inferior, apareciendo como un tubérculo adosado al aspecto distolingual de la corona de éste. ${ }^{2}$ Bamgbose y colaboradores ${ }^{14}$ hallaron sólo tres (3.4\%) casos de fusión con el tercer molar en 87 cuartos molares superiores e inferiores encontrados en un estudio de prevalencia. Asimismo, existen pocos reportes de casos clínicos en la literatura reciente sobre esta condición; sin embargo, la mayoría ha registrado la fusión con el tercer molar inferior. $5,6,17-20$

Debe diferenciarse a la fusión dental de la geminación y la concrescencia. La geminación es la división incompleta del folículo dentario al iniciarse el desarrollo de la corona que termina con la formación incompleta de dos órganos dentarios. La concrescencia es la fusión de dos órganos dentarios a nivel radicular, con depósito de cemento que acontece tras la formación completa de los mismos. ${ }^{2}$

La fusión del cuarto molar con un tercer molar inferior erupcionado parcial o completamente está asociada a una
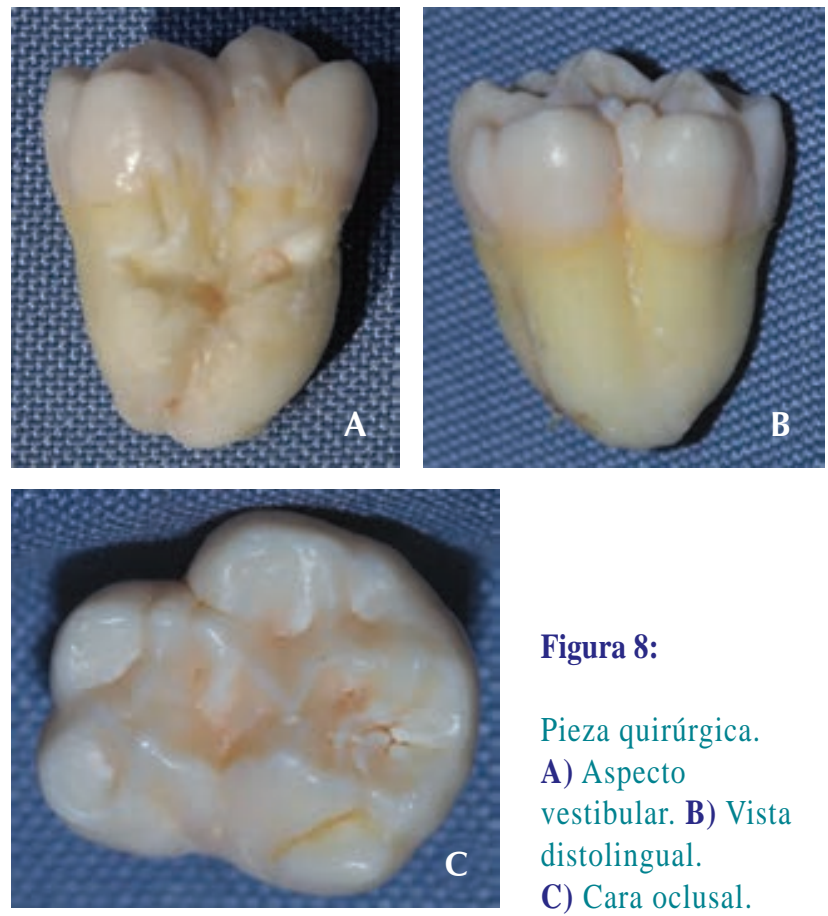

Figura 8:

Pieza quirúrgica.

A) Aspecto vestibular. B) Vista distolingual.

C) Cara oclusal.

alta predisposición para caries y enfermedad periodontal, y como resultado del proceso inflamatorio periodontal recurrente se puede desarrollar una pericoronitis o un quiste paradental. ${ }^{3,6}$

\section{CONCLUSIONES}

Como se ha podido observar, la fusión entre un órgano dentario normal con uno supernumerario es una situación clínica inusual, sobre todo tratándose de un diente supernumerario cuya frecuencia de aparición clínica es muy baja, como lo es la del distomolar inferior. El diagnóstico de esta condición se establece a través de la exploración clínica y la evaluación de estudios de imagen, el tratamiento consistirá casi invariablemente en la odontectomía.

\section{REFERENCIAS}

1. Sapp JP, Eversole LR, Wysocki GP. Patología oral y maxilofacial contemporánea. 2a ed. España: Elsevier Mosby; 2005.

2. Gay EC, Berini AL. Tratado de cirugía bucal. España: Ergon; 2004.

3. Ozcan G, Seckerci AE, Soylu E, Nazlim S, Amuk M, Avci F. Role of cone-beam computed tomography in the evaluation of a paradental cyst related to the fusion of a wisdom tooth with a paramolar: a rare case report. Imaging Sci Dent. 2016; 46 (1): 57-62.

4. Cunha RS, Junaid A, Mello I. Unilateral fusion of a supernumerary tooth to a maxillary permanent lateral incisor: a report of a rare case. J Endod. 2015; 41 (3): 420-423. 
5. Ferreira-Junior O, de Ávila LD, Sampieri MB, Días-Ribeiro E, Chen WL, Fan S. Impacted lower third molar fused with a supernumerary tooth-diagnosis and treatment planning using cone-beam computed tomography. Int J Oral Sci. 2009; 1 (4): 224-228.

6. Prakash AR, Reddy PS, Rajanikanth M. Paradental cyst associated with supernumerary tooth fused with third molar: a rare case report. J Oral Maxillofac Surg. 2012; 16 (1): 131-133.

7. Herrera-Atoche JR, Zúñiga-Herrera ID, Colomé-Ruíz GE, CarrilloÁvila BA. Distribución de dientes supernumerarios y anomalías dentales asociadas en una población ortodóntica del estado de Yucatán. Rev Odontol Latinoam. 2017; 9 (2): 49-55.

8. Jammal-Dobles N, Silva-Meza R. Prevalencia de dientes supernumerarios en una muestra mexicana. Rev Mex Ortodon. 2015; 3 (2): 88-91.

9. Núñez-Martínez JM, Sierra-Rayón J, García-López S, Tenorio-Torres G, Osorno-Escareño MC. Distribución y localización de los dientes supernumerarios en niños y adultos en una población mexicana: estudio radiográfico retrospectivo. Rev Cienc Clin. 2007; 8 (2): 59-63.

10. Salcido-García JF, Ledesma-Montes C, Hernández-Flores F, Pérez D. Garcés-Ortíz M. Frecuencia de dientes supernumerarios en una población mexicana. Med Oral Patol Oral Cir Bucal. 2004; 9 (5): 403-409.

11. Lu X, Yu F, Liu J, Cai W, Zhao Y, Zhao S et al. The epidemiology of supernumerary teeth and the associated molecular mechanism. Organogenesis. 2017; 13 (3): 71-82.

12. McBeain M, Miloro M. Characteristics of supernumerary teeth in nonsyndromic population in an urban dental school setting. J Oral Maxillofac Surg. 2018; 76 (5): 933-938.

13. Vázquez-Mosqueyra VM, Espinosa-Meléndez MT, HernándezFlores F. Presencia del cuarto molar. Revisión de la literatura. Rev Odont Mex. 2018; 22 (2): 104-118.
14. Bamgbose BO, Okada S, Hisatomi M, Yanagi Y, Takeshita Y, Abdu ZS et al. Fourth molar: a retrospective study and literature review of a rare clinical entity. Imaging Sci Dent. 2019; 49 (1): 27-34.

15. Casseta M, Altieri F, Giansanti M, Di-Giorgio R, Calasso S. Morphological and topographical characteristics of posterior supernumerary molar teeth: an epidemiological study on 25,186 subjects. Med Oral Patol Oral Cir Bucal. 2014; 19 (6): e545-e549.

16. Shahzad KM, Roth LE. Prevalence and management of fourth molars: a retrospective study and literatura review. J Oral Maxillofac Surg. 2012; 70 (2): 272-275.

17. Zeylabi A, Shirani F, Heidari F, Farhad AR. Endodontic management of a fused mandibular third molar and distomolar: a case report. Aust Endod J. 2010; 36 (1): 29-31.

18. López-Carriches C, Leco-Berrocal I, Baca Pérez-Bryan R. Fusión de un tercer molar mandibular con un cuarto molar supernumerario. Rev Esp Cir Oral Maxillofac. 2008; 30 (5): 344-347.

19. Hernández-Guisado JM, Torres-Lagares D, Infante-Cossío P, Gutiérrez-Pérez JL. Geminación dental: presentación de un caso. Med Oral. 2002; 7 (3): 231-236.

20. Conte M, Lombardi P, Linfant J. A supernumerary tooth fused to the distal surface of a mandibular third molar. J N J Dent Assoc. 2002; $73(1-2): 8-9$

\section{Correspondencia:}

Enrique Darío Amarillas Escobar

E-mail: dario_amarillas@hotmail.com

Conflicto de intereses: El autor declara no tener ningún conflicto de intereses.

Aspectos éticos: Ninguno.

Financiamiento: Ninguno. 